\title{
Spin liquid phase for the Frustrated Quantum Heisenberg Antiferromagnet on a square lattice
}

\author{
Jaime Ferrer \\ Serin Physics Laboratory, Rutgers University, P.O. Box 849, Piscataway, NJ 08855-0849
}

(Received April 1992)

\begin{abstract}
The existence of a spin disordered ground state for the frustrated $J_{1}-J_{2}-J_{3}$ Quantum Heisenberg Antiferromagnet on a square lattice is reconsidered. It is argued that there is a unique action which is continuous through the whole phase diagram, except at the Lifshitz point, so that the Neel and helicoidal states can not coexist and that there has to be an intermediate spin liquid state. To show it, a detailed study combining Spin-Wave Theory, Schwinger Bosons Mean Field Theory and a scaling analysis of the appropriate hydrodynamic action is performed. When done carefully, all these theories agree and strongly support the existence of the spin liquid state.
\end{abstract}

PACS numbers: 75.10.Jm,74.65.+n,75.50.Ee

\section{INTRODUCTION}

The past few years have seen a considerable effort in understanding the nature of the ground state and excitations of low-dimensional Quantum Antiferromagnets, due to its close link to high- $T_{c}$ superconductors [1, 2, 3, , 1 . One of the most fundamental discussed issues is the existence and properties of spin liquid states. [5, 6, 76].

The way in which one can induce a spin disordered state in a magnet is by increasing fluctuations about the ordered state, for instance, by adding frustration to the bare Heisenberg model -antiferromagnetic coupling constants to next to nearest neighbors- $\$$. We study in this paper the phase diagram of the quantum $J_{1}-J_{2}-J_{3}$ model at zero temperature (all $J_{i} \geq 0$ ). We show in figure 1 the phase diagram of the corresponding classical model. 9]. We see that there are four possible states -Neel, collinear and two helicoidal- separated by boundaries. The transition between Neel and collinear states is discontinuous because they have different symmetry. The same happens in the boundary between the $(q, q)$ and $(q, \pi)$ helicoidal states. The other transitions (marked with dashed lines) are continuous and separate states of the same symmetry. The Classical Critical Line (CCL) $J_{1}-2 J_{2}-4 J_{3}=0$ is the separatrix between Neel and helicoidal states, and its endpoint, $J_{3}=0$, is a Lifshitz point.

The first studies on the phase diagram of the quantum model were performed by Chandra and Doucot [8] (for $\left.J_{3}=0\right)$ using Linear Spin Wave Theory (LSWT) and by Ioffe and Larkin 10, who made a Renormalization Group (RG) analysis of a generalized nonlinear $\sigma$ model. 
Both of them concluded that the combined effect of quantum fluctuations and frustration were strong enough to destroy long-range-order (LRO) along some critical line yielding a spin liquid state. Later on, Moreo et al [9] and Chubukov 11] generalized the LSWT analysis to $J_{3} \neq 0$ (see figure 1). This scenario was supported by series expansion [12], finite-size diagonalizations [13, 14 (for $J_{3}=0$ ) and a momentum-shell RG study [15]. On the other hand, several mean field-BCS-type theories of interacting bosons have challenged this result, either for $J_{3}=0$ [16, 17, 18, 19] or for the general case [20]. They predict that the stability of the Neel state is enhanced over the classical case due to quantum fluctuations (Villain's order from disorder [21]) along the whole critical line, while that of the spiral state is not modified in an essential way.

The purpose of this paper is to clarify this controversy. Our main points will be:

1. The $C C L$ separates states with the same symmetry. The transition is continuous classically, in the sense that the parameters of the long wavelength action $\mathcal{S}\left[J_{i}\right]$ vary smoothly along the transition. We argue that the same happens for the quantum case, $\mathcal{S}\left[J_{i}, S\right]$. In particular, we show that, although there is an enhancement of the stability of the Neel state close to the $C C L$, there is a bigger decrease of the stability of the spiral state. This means that the $C C L$ is continued in a Quantum Critical Plane, $Q C P$, which depends on $\mathrm{S}$ and is tilted upwards with respect to the plane $J_{1}-2 J_{2}-4 J_{3}$ (see figure $1)$.

2. Because the quantum action is continuous through the $Q C P$, we can use a generalized Sigma model to describe the physics of the transition along the $Q C P$ and at its Neel side. We evaluate that action along the $Q C P$ using a Renormalization Group analysis and find that the relevant coupling constant always flows to strong coupling meaning that the spin state is disordered. We then proceed to show that the spin liquid state exists not only along that plane but also in a finite region around it. As a corollary, we link this scaling analysis for the strongly frustrated magnet with the one performed in reference 2 , for the weakly frustrated case.

3. The end point of the $C C L$ is a Lifshitz point. In this case, the counterpart of the Neel state is not spiral, but collinear. Because both states have different symmetry, the quantum action for both states is different, and therefore we cannot establish any continuity principle. We discuss this case in the last section. 


\section{MICROSCOPIC STUDY OF THE $J_{1}-J_{2}-J_{3}$ MODEL}

The purpose of this section is to study the stability diagram of the quantum $J_{1}-J_{2}-J_{3}$ model around the $C C L$. Because the whole line has the same physics, we can concentrate our analysis just to one, representative point of the phase diagram. We will choose the plane $J_{2}=0$ for simplicity, in which case the $C C L$ is $J_{3}=0.25 J_{1}$. We will work in units in which $J_{1}=1$.

The energy of the classical $J_{1}-J_{2}-J_{3}$ model is given by

$$
E=\sum_{<i, j>} J_{i j} \vec{S}_{i} \cdot \vec{S}_{j}=\frac{S^{2}}{2} J_{Q_{0, c l}}
$$

where $J_{Q}$ is the Fourier transform of $J_{i j}$ and $\vec{Q}_{0, c l}$ is the pitch wave vector of the spiral state, which is obtained by minimizing the energy: $\partial_{Q} J_{Q_{0, c l}}=0 . \vec{Q}_{0, c l}$ is a continuous function of $J_{2} / J_{1}$ and $J_{3} / J_{1}$, with a jump in its derivative at the critical value $J_{1}-2 J_{2}-4 J_{3}=0$. This equation defines the Classical Critical Line. The classical spin stiffness is given by

$$
\rho_{i j}^{z}=\left.\frac{\partial^{2} E}{\partial Q_{i} \partial Q_{j}}\right|_{\vec{Q}_{0, c l}}
$$

and it also varies continually with $\vec{Q}_{0, c l}$.

We can then write the most general action for an helical magnet by noting that the order parameter space is $O(3) \times U(1) / U(1), 22,23,24$

$$
\mathcal{S}=-\frac{1}{2} \int d^{2} x \operatorname{Tr}\left\{A_{i} P_{i} A_{i}\right\}=-\frac{1}{2} \int d^{2} x \rho_{a}\left(A_{i}^{a}\right)^{2}
$$

where $A_{i}=A_{i}^{a} T_{a}=g^{-1} \partial_{i} g$ is a pure gauge field, $g(x) \epsilon S O(3)$ and $T_{a} \in \operatorname{Lie}[S O(3)]$. The gauge field is equivalent to a twist of the order parameter and serves to define the spin stiffnesses, $\rho_{a}=\left(\rho_{x}, \rho_{x}, \rho_{z}\right)$. The action $S\left[\rho\left(\vec{Q}_{0, c l}\right)\right]$ is unique for both Neel and helical states and the spin stiffnesses are continuous throughout the whole phase diagram.

We argue that, after introducing quantum fluctuations, there is still a unique action for the whole phase diagram, which varies continually when passing from the Neel to the spiral state through the Quantum Critical Plane. The action picks up an extra piece due to the fact that the fields become dynamical and an implicit dependence on S through $\vec{Q}_{0}(S)$ :

$$
\mathcal{S}=-\frac{1}{2} \int d x_{0} d^{2} x\left(\chi_{a}\left(A_{0}^{a}\right)^{2}+\rho_{a}\left(A_{i}^{a}\right)^{2}\right)
$$

All this implies that if the stability of the Neel state gets enhanced due to quantum fluctuations, (1) the pitch wave vector of the helical state also renormalizes so as to adjust smoothly to the new boundary of the Neel state, 
where its value is $(\pi, \pi),(2)$ the spin stiffness of the helical phase is equally decreased, so that both go to zero at the same point. To substantiate this ideas, we will perform in this section a Spin Wave Theory (SWT) study of the magnetization, the pitch wave vector and the spin stiffness and complement it, eventually with results from Schwinger Bosons Mean Field Theory (SBMFT). Details of the derivation and notation are relegated to appendices $\mathrm{A}$ and $\mathrm{B}$.

As a starting point, let's determine the phase diagram from LSWT and SBMFT. The magnetization for the helicoidal $\overrightarrow{Q_{0}}=\left(q_{0}, q_{0}\right)$ and Neel $\overrightarrow{Q_{0}}=(\pi, \pi)$ states from LSWT is

$$
<S^{z}>==S+\frac{1}{2}-\frac{1}{2 N} \sum_{k} \frac{J_{k}+J_{ \pm}-2 J_{Q_{0}}}{E(k)}
$$

$\overrightarrow{Q_{0}}$ is obtained by minimizing the ground state energy: $\cos \left(q_{0}\right) \sim \cos \left(q_{0, c l}\right)=-\frac{1}{4 J_{3}}$ (for the Neel state, $\left.\cos \left(Q_{0}\right)=-1\right)$. The denominator is the energy of the spin waves. The value of $\mathrm{S}$ for which the staggered magnetization $\left\langle S^{z}>\right.$ is zero determines the boundaries of the long range ordered states.

We plot in figure 2 the stability diagram we have obtained using LSWT and SBMFT. The theories are in disagreement: (1)LSWT predicts a decrease of the stability of both ordered states, with a line of second order phase transitions ending at the critical point; (2) SBMFT predicts an enhancement of the stability of the Neel state. This enhancement is also seen in numerical diagonalization studies [9]. On the other hand, the boundary of the spiral state doesn't move towards the right as we introduce quantum fluctuations. This is a spurious result, due to the fact that large- $\mathrm{N}$ theories don't give the correct Goldstone mode structure for the spin wave spectrum. As a result, we obtain a first order phase transition.

Let's concentrate for a moment on the Neel state and compute the expression for the staggered magnetization close to the classical frustrated point. Evaluating the leading divergences we obtain

$$
<S^{z}>\simeq S+\frac{1}{2}-\alpha \ln \left(\rho_{c l}\right)+\frac{\beta}{S} \frac{1}{\rho_{c l}}+O\left(\frac{1}{S^{2}}\right)
$$

where $\rho_{c l}=J_{1}-4 J_{3}$ is the classical spin stiffness which we use here as a cutoff for the infrared divergent integrals. This series can be resumed: computing $\mathcal{E}(k)$-the spin wave energy to next to LSWT order in $1 / S-$, we find:

$$
\begin{aligned}
<S^{z}> & \simeq S+\frac{1}{2}-\gamma \ln (\rho(S)) \\
\rho(S) & \simeq \rho_{c l}-\frac{4 J_{3}}{S}\left(\alpha_{3}-\alpha_{1}\right)
\end{aligned}
$$

which is the LSWT result with a renormalized spin stiffness. It serves us to define the $Q C P$, i.e.: the line where the spin stiffness, computed to next to LSWT order is zero. As shown in figure $2, \rho(S)$ is tilted to the right of the classical value, with a slope $\sim 5.2$. This fact supports 
the order from disorder conjecture from the point of view of SWT.

Based in our symmetry argument, we argue that this line has to be the same for both the helicoidal and the Neel states (at least for large $\mathrm{S}$ ). In other words, the enhancement of the stability of the Neel state is accompanied by a similar reduction of the stability of the helicoidal one so that both states match each other continuously. To prove it, we compute the LSWT correction to the pitch wave vector and to the stiffness:

$$
\begin{aligned}
q_{0}^{i}-q_{0, c l}^{i}= & \Delta q_{0}^{i}=\frac{1}{2 S \partial_{i}^{(2)} J_{Q_{0}, c l}} \sum_{k} \partial_{i} J_{ \pm}\left(\frac{J_{k}-J_{Q_{0}, c l}}{J_{ \pm}-J_{Q_{0, c l}}}\right)^{1 / 2}+O\left(\frac{1}{S^{2}}\right) \\
\rho_{i i}^{z}= & \frac{S^{2} \partial_{i}^{(2)} J_{Q_{0, c l}}}{2} \\
& +\frac{S}{2}\left\{\partial_{i}^{(2)} J_{Q_{0, c l}}-\left(\sin \left(q_{0, c l}\right)+8 J_{3} \sin \left(2 q_{0, c l}\right)\right) S \Delta q_{0}^{i}\right. \\
& \left.+\frac{1}{2} \sum_{k} \frac{\left(2 J_{Q_{0, c l}}-J_{k}-J_{ \pm}\right) \partial_{i}^{(2)} J_{Q_{0, c l}}-\left(J_{Q_{0, c l}}-J_{k}\right) \partial_{i}^{(2)} J_{ \pm}}{E(k)}\right\}+O(1)
\end{aligned}
$$

The spin stiffness of the unfrustrated magnet is $\rho=$ $\rho_{c l}\left(1-\frac{0.118}{S}\right)\left(=0.764 \rho_{c l}\right.$ for spin $\left.1 / 2\right)$. This estimate is in accordance with that obtained using the renormalization of the spin wave velocity and the susceptibility [2], $\rho=$ $\rho_{c l} Z_{\chi} Z_{c}^{2} \simeq \rho_{c l}\left(1-\frac{0.276}{S}\right)\left(1+2 \times \frac{0.079}{S}\right)$.

We draw in figure $3\left(\right.$ a) the LSWT correction to $q_{c l}$ for the spiral state to show that it matches with $(\pi, \pi)$ continually, although the boundary of stability of the Neel state be shifted to the right. Figure $3(\mathrm{~b})$ is a plot of the LSWT correction to the spin stiffness, and shows that it is enhanced for the Neel state close to the critical point, signaling the order from disorder phenomenon. The important point is that it is decreased by a bigger amount on the other side. We stress that this beautiful results are already obtained in LSWT.

Before entering in the next section, we would like to summarize the most important conclusions we have obtained so far. We can very safely state that the stability of the Neel state is enhanced over the classical values over the whole critical line, and that this is due to the fact that the spin stiffness $\rho(S)$ gets renormalized upwards due to quantum fluctuations, so that it defines a $Q C P$ tilted to the right of the $C C L$. The stability of the helicoidal state is reduced in the same way, so that both states match smoothly and the long wavelength physics is continuous through the transition point. Because of that, a modified $\sigma$ model which describes the spin wave excitations of the strongly frustrated Neel state is appropriate to study the $Q C P$ and its neighborhood. These conclusions don't apply to the $J_{1}-J_{2}$ model due to the fact that in that case the two states have different symmetry and the spin stiffnesses close to the tricritical point are different. 


\section{SCALING ANALYSIS}

We devote this last section to perform a Renormalization Group study of the hydrodynamic action appropriate for the strongly frustrated magnet. It is a generalization of the $O(3) / U(1)=S^{2}$ quantum non linear $\sigma$ model. We will review in section 4.1 well-known results for the conventional $O(3)$ case, published by other authors, which are pertinent to our discussion. In section 4.2 , we pass to the strongly frustrated case, and show that along the $Q C P$ the system always flows towards strong coupling. The results of this section complement our previous findings from microscopic models and give us a very consistent picture of the phase diagram of the $J_{1}-J_{2}-J_{3}$ model.

\section{A. Unfrustrated case}

It is generally believed that the long-wavelength action of a non-frustrated Quantum Antiferromagnet in d dimensions is given by a $(d+1)$-dimensional non-linear $\sigma$ model [25]

$$
\begin{aligned}
\mathcal{S} & =\frac{\rho^{0}}{2} \int_{-\infty}^{\infty} d t \int_{a} d^{d} r\left\{(\nabla \hat{n})^{2}-\frac{1}{c_{0}^{2}}\left(\partial_{t} \hat{n}\right)^{2}\right\} \\
& =\frac{1}{2 g_{0}} \int_{-\infty}^{\infty} d \tilde{t} \int_{1} d^{d} \tilde{r}\left\{(\nabla \hat{n})^{2}-\left(\partial_{\hat{t}} \hat{n}\right)^{2}\right\}
\end{aligned}
$$

where $\tilde{r}=r / a, \tilde{t}=t c / a$ are dimensionless variables, $\rho_{0}=J_{1} S^{2} a^{2-d}$ is the bare spin stiffness, $c_{0}=2 \sqrt{d} J_{1} S a$ is the spin wave velocity and

$$
g_{0}=\frac{c_{0}}{\rho_{0} a^{d-1}}=\frac{1}{\sqrt{\rho_{0} \chi_{0} a^{d-1}}}=\frac{2 \sqrt{d}}{S}
$$

is the bare coupling constant. All these magnitudes are defined at the scale of the lattice cutoff, a.

In $1+1$ dimensions, one has to add to this action a topological $\theta$ term, which distinguishes between integer and half-integer spins [25]. It has been proven that such a term gives a zero contribution in $2+1$ dimensions for any topological configuration of the n-fields [26,27,28]. Moreover, as long as the n-field is continuous at all points no Hopf term appears in the action. Once this condition is relaxed, though, that term appears and affects the physics of the system 26,29]. Such a situation could arise if the $\sigma$ model were in its disordered phase.

One of the main issues concerning the nonlinear quantum $\sigma$ model is the connection with the Classical one, which is much better known. This connection can be done in principle when the contribution of the Berry phase is zero. Strictly speaking, the mapping is rigorous only for large $\mathrm{S}$ and long wavelengths. We therefore face two problems when we try to link it with the microscopic quantum models of interest: 
- We don't know by certain if the action is still valid in the extreme quantum limit. In this respect, nevertheless, we can argue that it gives the correct spin wave spectrum and interaction between spin waves at long wavelengths, as one can check by calculating the staggered magnetization, spin wave energy and damping,... in the first few orders of perturbation theory

- A more difficult point is the identification of the parameters entering in the action in terms of the ones appearing in the microscopic model. The problem is that the values obtained in the derivation are well suited only for large spin and long wavelength scales, and they don't have to hold necessarily for small S. In fact, they don't. One possibility is to connect those values with the ones obtained by SWT. But it is not clear which order in $1 / S$ should one use as input of the continuum model. That should depend on how strongly the Goldstone modes interact and, therefore, on the dimension.

Polyakov pioneered the work on the Classical NL $\sigma \mathrm{M}$ by recognizing that close to its lower critical dimension Goldstone modes interact strongly and become critical. He proceed then to solve the problem using a Renormalization Group approach. Later on, the same problem was addressed by Brézin and Zinn-Justin 31] in the context of the $\epsilon$-expansion $(\epsilon=d-2)$ to order $\epsilon^{2}$ and by Nelson and Pelcovits [32] using momentum-shell recursion relations [33] to one loop order, i.e.: to first order in $\epsilon$. The approximation is expected to be very good in two dimensions, but it is not so clear that this is the case in three (where $\epsilon=1$ ).

Once we have an action to describe the system in $2+1$, we have to distinguish between short wavelength quantum critical fluctuations and the long wavelength antiferromagnetic magnons. The crossover length between both is the Josephson correlation length $\xi_{J}$ [34. $\xi_{J}$ tells us the extent to which the quantum model can be mapped to the classical one. This problem was addressed by Chakravarty, Halperin and Nelson in an important publication [2]. CHN combined the techniques of Nelson and Pelcovits with the method developed by Hertz [35] to deal with quantum critical phenomena at finite temperatures in two spatial dimensions. They showed that:

- At $T=0$ the model is equivalent to a 3 -dimensional classical one where $g \sim 1 / S$ plays the role of the temperature. In that case, the $\mathrm{QNL} \sigma \mathrm{M}$ has a phase transition from a Neel ordered to a (quantum) disordered state, at a value $g_{c}$ of the coupling constant. The Josephson correlation length turns out to be of the order of a few lattice spacings for $g<g_{c}$, but grows with $g$ until it diverges at $g_{c}$.

- For $g>g_{c}$ the system is in a quantum disordered phase. In that phase, CHN obtain $\xi \sim \frac{S a}{S_{c}-S}$ for the correlation length. It is very likely that instanton 
contributions to the Hopf term will stabilize spinPeierls order here [29].

- At finite, but not too large, temperatures in the region where the ground state is Neel ordered $(g<$ $g_{c}$ ) we can integrate all quantum fluctuations to map the QNL $\sigma \mathrm{M}$ into a classical problem with a renormalized spin stiffness and a short wavelength cutoff of order $\hbar c / k_{B} T$.

Our goal is to analyze the stability of the Neel state when introducing frustration. We will therefore use CHN approach in the zero temperature formalism. To begin with, we recalculate some of the magnitudes already discussed by the former authors. We defer to appendix C the derivation of the scaling equations, and quote here only the results. We obtain:

$$
\begin{aligned}
& \frac{d \bar{g}}{d l}=-\bar{g}+\bar{g}^{2} \\
& \frac{d \rho}{d l}=-\rho \bar{g} \\
& \frac{d \sigma}{d l}=\sigma \bar{g} \\
& \bar{g}_{0}=\frac{S_{c}}{S}=\frac{0.225}{S}
\end{aligned}
$$

where $\sigma$ is the component of $\hat{n}$ along the quantization axis and $\bar{g}=\frac{g}{4 \pi}$. Integrating

$$
\begin{aligned}
\bar{g}_{l} & =\frac{\bar{g}_{0} e^{-l}}{1-\bar{g}_{0}\left(1-e^{-l}\right)} \\
\rho_{l} & =\rho_{0}\left(1-\bar{g}_{0}\left(1-e^{-l}\right)\right) \\
\sigma_{0} & =\sigma_{\infty}\left(1-\bar{g}_{0}\right)
\end{aligned}
$$

We plot the flow diagram in figure 4(a). There are two different phases, separated by the straight line $g=$ 1. To the left of this separatrix $(g<1)$, we have the renormalized classical phase, where there is Neel long range order renormalized by quantum fluctuations. All the flow lines end at the $\rho$ axis, which is a line of fixed points. The solution of the scaling equations is:

$$
\begin{aligned}
g_{\infty} & =0 \\
\rho_{\infty} & =\rho_{0}\left(1-g_{0}\right)=\rho_{0}\left(1-\frac{S_{c}}{S}\right) \\
<\sigma>_{0} & =1-g_{0}=1-\frac{S}{S_{c}} S<\sigma>_{0}=S-S_{c}
\end{aligned}
$$

At the right of the separatrix, we have the quantum disordered phase, where interaction between magnons is so strong that confine them, generating an internal length scale $l^{*}=\ln \left(\frac{g_{0}}{g_{0}-1}\right)$ in terms of which we define the correlation length

$$
\xi \sim a e^{l^{*}}=\frac{g_{0} a}{g_{0}-1}=\frac{S_{c} a}{S_{c}-S} \stackrel{\stackrel{S \rightarrow 0}{\rightarrow}}{\stackrel{S \rightarrow S_{c}^{-}}{\rightarrow}} a
$$


The coupling constant $\mathrm{g}$ diverges and the spin stiffness goes to 0 at that length scale.

Finally, we think it is worth remarking that although figure 4(a) shows flow lines for $\rho \sim 0$, that result is spurious, because in that case the action for the QNL $\sigma \mathrm{M}$ is empty and one has to take into account quartic terms in the gradient expansion of the Heisenberg Hamiltonian.

\section{B. $J_{1}-J_{2}-J_{3}$ Model}

We are now in a position to understand better the strongly frustrated case. We will use a modified $\sigma$ model appropriate for both the weakly and strongly frustrated magnets in the Neel state. We will try to discern if the coupling constant flows towards strong coupling along the $Q C P$, signaling that the magnet is disordered for whatever the value of the spin.

Close to the $Q C P$ the bare spin stiffness, $\rho(S)$, is very small and we are forced to take into account effects due to quartic terms, just as we did in our calculation of the staggered magnetization in SWT. The action is

$$
\begin{aligned}
\mathcal{S}= & \left.\frac{1}{2} \int_{-\infty}^{\infty} \int_{a}^{L} d t d^{2} r\left\{\chi^{0}\left(\partial_{t} \hat{n}\right)^{2}-\rho^{0}(S)\left(\left(\partial_{x} \hat{n}\right)^{2}\right)+\left(\partial_{y} \hat{n}\right)^{2}\right)\right) \\
& \left.+\frac{\sigma_{1}^{0} a^{2}}{12}\left(\left(\partial_{x x} \hat{n}\right)^{2}+\left(\partial_{y y} \hat{n}\right)^{2}\right)-\sigma_{2}^{0} a^{2}\left(\partial_{x x} \hat{n}\right)\left(\partial_{y y} \hat{n}\right)\right\}
\end{aligned}
$$

We take $\rho^{0}(S)$ as given by SWT (i.e.: the stiffness renormalized by "short" wavelength quantum fluctuations), instead of $\rho_{0}=\left(J_{1}-2 J_{2}-4 J_{3}\right) S^{2}$, and use the values obtained in the gradient expansion for the rest of the parameters entering in the action:

$$
\begin{aligned}
\sigma_{1}^{0} & \sim\left(J_{1}-2 J_{2}-16 J_{3}\right) S^{2} \\
\sigma_{2}^{0} & \sim J_{2} S^{2} \\
g^{0} & =\frac{1}{\sqrt{\rho(S) \chi a}}=\sqrt{\frac{8 J_{1}}{J_{1}-2 J_{2}-4 J_{3}}} \frac{1}{S}
\end{aligned}
$$

We can already see one of the problems which arise in the frustrated case: the coupling constant depends not only on $\mathrm{S}$ (as in the unfrustrated case), but also on the exchange interactions $J_{1}, J_{2}$ and $J_{3}$. Therefore, we don't have such a clear indicator about the magnetic order of the microscopic model as before. Moreover, $g_{0}$ diverges at the $Q C P$, and therefore is not well-suited for our analysis. This means that we will have to cook a charge which doesn't contain $\rho(S)$ in the denominator.

After performing the perturbative RG expansion we arrive at the following scaling equations:

$$
\begin{aligned}
\frac{d g}{d l} & =-g+g^{2} I\left(1+\frac{\sigma_{1}-2 \sigma_{2}}{8 \rho}\right) \\
\frac{d \rho}{d l} & =-\rho g I\left(1+\frac{\sigma_{1}-2 \sigma_{2}}{4 \rho}\right) \\
\frac{d \sigma_{i}}{d l} & =-\sigma_{i}(2+g I)
\end{aligned}
$$




$$
\frac{d c}{d l}=-c g I \frac{\left(\sigma_{1}-2 \sigma_{2}\right)}{8 \rho}
$$

where $\mathrm{I}$ is the loop integral

$$
I=\frac{\sqrt{6 \rho}}{\pi^{2}} \int_{0}^{\pi / 2} \frac{d \theta}{\sqrt{24 \rho-2 \sigma_{1}+\left(\sigma_{1}+6 \sigma_{2}\right) \sin ^{2} \theta}}
$$

This integral diverges at the Lifshitz point, signaling that the scaling analysis breaks down there. We see that this point is quite pathological, and has to be treated in a different way from the rest of the phase diagram. We have written down the scaling equation for $c$ to show that in the frustrated case, it does get renormalized downwards. These scaling equations reduce to those obtained by $\mathrm{CHN}$ for the unfrustrated case and to those of Ioffe and Larkin when $\rho(S)=0$.

We will pass on now to the task of finding an unambiguous indicator of the ordering of the system. We have two possibilities to obtain a charge which roughly go like $1 / S$ and which give us the separatrix between the ordered and disordered states:

1. We can choose $\mathcal{G}_{0} \sim \frac{1}{\sqrt{\rho}}$. This kind of charges diverge if $\rho_{0}(S)$ is close to the $Q C P$. They have a typical behavior $\mathcal{G} \sim-\alpha(\rho) \mathcal{G}+\beta(\rho) \mathcal{G}^{2}$, which means that there is a separatrix but, because $\mathcal{G}_{0}>>1$, one is always in the disordered phase.

2. $\mathcal{G}_{0} \sim \frac{1}{\sqrt{\sigma_{1} \sigma_{2}}}$. In this case, the system always flows towards strong coupling and there is no separatrix.

There is a third possibility: we can evaluate the action strictly along the $Q C P$ and define, following Ioffe and Larkin [10], the following charge:

$$
G^{0}=\frac{I}{\sqrt{\rho(S)} \chi a}=\frac{A}{\chi^{1 / 2} \sigma_{1}^{1 / 4} \sigma_{2}^{1 / 4} a} \sim \sqrt{\frac{J_{1}}{\left(J_{2} J_{3}\right)^{1 / 2}}} \frac{1}{S} \quad\left(\rho^{0}=0\right)
$$

We then assume that $A=\left(\sigma_{1} \sigma_{2}\right)^{1 / 4} I / \rho^{1 / 2}(S)$ doesn't get renormalized. We have checked that the $Q C P$ is stable under iteration of the scaling equations. We find that G satisfies:

$$
\frac{d G}{d l}=G^{2} \quad: G_{l}=\frac{G_{0}}{1-G_{0}\left(l-l_{0}\right)}
$$

It flows towards strong coupling, which means that it stays effectively at its lower critical dimension, with the generation of a correlation length $\xi \sim a e^{\frac{1}{\varepsilon_{0}}} \sim a e^{S}$.

We should draw now the separatrix between the ordered state and the spin liquid. Ioffe and Larkin argue that for finite $\rho(S)$ the relevant action is not given by equation 14 , but by

$$
\begin{aligned}
\mathcal{S}= & \frac{1}{2} \int_{-\infty}^{\infty} \int_{\ln \left(\frac{1}{\rho(S)}\right)}^{L} d t d^{2} r\left\{\chi^{0}\left(\partial_{t} \hat{n}\right)^{2}\right. \\
& \left.+\frac{\sigma_{1}^{0} a^{2}}{12}\left(\left(\partial_{x x} \hat{n}\right)^{2}+\left(\partial_{y y} \hat{n}\right)^{2}\right)-\sigma_{2}^{0} a^{2}\left(\partial_{x x} \hat{n}\right)\left(\partial_{y y} \hat{n}\right)\right\}
\end{aligned}
$$


where $\ln \left(\frac{1}{\rho(S)}\right)$ acts as a cutoff in the scaling equations. In that case, the separatrix is given by

$$
1+G_{0} \ln (\rho(S))=0
$$

We stress that Ioffe and Larkin's action is different from the naive hydrodynamic one, equation 14. It is not clear to us which one of them really describes the physics of the $J_{1}-J_{2}-J_{3}$ model close to the $Q C P$. In any case, there is no doubt that the separatrix has to exist, be it an exponential or not. We show in figure 4(b) the flow diagram of the complete $J_{1}-J_{2}-J_{3}$ model, where we draw a tentative separatrix for small $\rho$ and join it with the one given for the weakly frustrated case. It is amusing to note that turning the figure by $90^{\circ}$, the diagram is the same as the one given by SWT and SBMFT, if we take into account that we are writing the dressed $\rho(S)$ in the ordinate axis.

\section{Discussion}

We recapitulate here the main conclusions we have reached:

- We have found that the system is at its lower critical dimension along the $Q C P$ : the charge flows to strong coupling, signaling a spin liquid state.

- There is a separatrix between the spin liquid phase and the Neel ordered state. That line begins at the point $(\rho(S), g)=(0,0)$ and links the CHN separatrix for sufficient large values of the spin stiffness.

- The flow diagram looks very much like the stability diagram that we obtained from SWT and SBMFT, with an enhancement of the Neel phase due to order from disorder.

- There is a dimensional crossover from the region $\rho(S)<<1$, where the system is $2+1$ dimensional, and $g^{\prime}=-g+g^{2}$ to the region where the spin stiffness is very small, and the system is at its critical dimension, $d_{c}$, so that $G=G^{2}$.

\section{IV. $J_{1}-J_{2}$ MODEL}

We devote this section to study the $J_{1}-J_{2}$ model. We have already argued that it is the most complicated case of the whole phase diagram, and probably, the least relevant. We nevertheless study it because it has attracted a lot of attention.

We begin, as in section II, by plotting the phase diagram obtained from LSWT and SBMFT (figure 5(a)). The expressions for the magnetization of the Neel and collinear states from LSWT as a function of $J_{2} / J_{1}$ are [8]: 


$$
\begin{aligned}
<S^{z}> & =S+\frac{1}{2}-\frac{1}{N} \sum_{k} \frac{1-J_{2}\left(1-\Gamma_{k}\right)}{\sqrt{\left(1-J_{2}\left(1-\Gamma_{k}\right)\right)^{2}-\gamma_{k}^{2}}} \quad(\text { Neel }) \\
& =S+\frac{1}{2}-\frac{1}{N} \sum \frac{1+\lambda \cos \left(k_{y}\right)}{\sqrt{\left(1+\lambda \cos \left(k_{y}\right)\right)^{2}-\cos \left(k_{x}\right)^{2}\left(\lambda+\cos \left(k_{y}\right)\right)^{2}}}(\mathrm{col})
\end{aligned}
$$

where $\lambda=1 / 2 J_{2}$. The denominators are the velocities of the spin waves. The value of $\mathrm{S}$ for which the magnetization $<S^{z}>$ goes to zero defines the boundary we are looking for.

As already stated by other authors [17,18], LSWT predicts a finite region where the ground state is disordered around the classical tricritical point $J_{2}=0.5$ for any $\mathrm{S}$ (and in particular, for $S=\frac{1}{2}$ ). On the contrary, mean field theories predict that the stability of the Neel state is enhanced by quantum fluctuations with a reentrant boundary joining the classical frustrated point with zero slope. This fact means that there is, ineludibly, a region of coexistence of Neel and collinear states and therefore, a finite hysteresis and a first order phase transition. This first order phase transition extends even for $S<1 / 2$, so that there isn't a spin liquid state for spin one-half.

We would like to devote some words to some interesting features of the SBMFT boundary of the collinear state. In fig. 5(b) we show the SBMFT results in a logarithmic scale. It shows a bump where the stability of the collinear state is even bigger than that of the unfrustrated case and then tends to the correct result for $J_{2} \rightarrow \infty(1 / S \sim 5.1)$. In the same figure we show the results obtained in numerical diagonalizations 13] for spin one-half. We see that the boundaries of the ordered states from mean field theory are much higher than the results of numerical diagonalizations. These two pieces of evidence support our belief that mean field theories overestimate the stability of the ordered states and that there is a spin liquid in a very small region at spin $1 / 2$ for $J_{2} \sim 0.6$.

We solve now the discrepancy between LSWT and SBMFT. We evaluate the staggered magnetization of the Neel state in the next to LSWT order in $1 / S$ close to the $C C L$ :

$$
<S^{z}>\simeq S+\frac{1}{2}-\alpha \ln ^{2}\left(\rho_{c l}\right)+\frac{\beta}{S} \frac{\ln ^{2}\left(\rho_{c l}\right)}{\rho_{c l}}+O\left(\frac{1}{S^{2}}\right)
$$

where we use $\rho_{c l}=J_{1}-2 J_{2}$ as a cutoff for the infrared divergent integrals and $\alpha$ and $\beta$ are constants. This series can be resumed by computing $\mathcal{E}(k)$, the spin wave energy, to next to LSWT order in $1 / S$. Using the spin wave velocity

$$
\rho(S) \sim \rho_{c l}-\frac{2 J_{2}}{S}\left(\alpha_{2}-\alpha_{1}\right)
$$

as a cutoff, instead of the spin stiffness, we find:

$$
<S^{z}>\simeq S+\frac{1}{2}-\gamma \ln ^{2}(\rho(S))
$$

which means that $1 / S$ corrections to LSWT just renormalize the CCL where the spin stiffness (and spin wave 
velocity) is zero. The $Q C P$ for the Neel state along which the spin stiffness is zero gets tilted to the right as one goes further in perturbation theory in $1 / S$. Therefore, SWT also predicts an enhancement of the stability of the Neel state and therefore, order from disorder. In other words: it tends to coincide with SBMFT.

An interesting feature of equations 22 and 23 is that $<S^{z}>$ diverges as the square of the logarithm of the cutoff, signaling that the Renormalization Group analysis does not work at that point.

\section{CONCLUSION}

The purpose of this paper was to shed some new light on the recent controversy about the existence of a spin liquid phase in the phase diagram of the $J_{1}-J_{2}$ and $J_{1}-J_{2}-J_{3}$ models. We have tried to do a thorough analysis of the problem using different techniques, comparing them and extracting consequences about the validity of each one. We have obtained a very consistent qualitative picture of how the phase diagram looks like. For the $J_{1}-J_{2}$ model, there seems to be a first order phase transition, due to the fact that the stability of the Neel state is enhanced by quantum fluctuations. At the same time there probably exists a very small region where the magnet is disordered for spin $\frac{1}{2}$. Its frustrated point, though, is a Lifshitz point and therefore is not representative of the phase diagram of the $J_{1}-J_{2}-J_{3}$ model. In the $J_{1}-J_{2}-J_{3}$ model we have found order from disorder, too. But now there is a finite region where the system is disordered for any value of the spin, because the stability of the helicoidal phase is reduced as much as the one of the Neel phase is enhanced, and there is no first order phase transition. For spin $\frac{1}{2}$ the spin liquid region is shifted from the Classical Critical line $J_{1}-2 J_{2}-4 J_{3}=0$ (figure 1 ) to considerably bigger values of $J_{2}$ and $J_{3}$. We find that the character of the spin disordered phase seems to be different for the weakly and for the strongly frustrated cases. This fact could have any repercussion in the role of instanton tunneling between different topology sectors, and the stabilization of spin-Peierls order close to the critical line. Finally, we would like to mention that our results agree with numerical diagonalization studies by Dagotto et al [9, 13] both for the $J_{1}-J_{2}-J_{3}$ and the $J_{1}-J_{2}$ models but are in contradiction with the recent work of Schulz and Ziman [14 and with the results obtained from series expansions [12] for the $J_{1}-J_{2}$ model.

\section{ACKNOWLEDGMENTS}

We would like to thank P. Coleman, L. Ioffe, F. Gebhard, N. Andrei, H. Neuberger, M. Bèzard, X. Y. Zhang, P. Chandra and F. Mila for illuminating discussions. The 
financial support from the Fulbright-MEC (Spain) program is also gratefully acknowledged.

\section{APPENDIX A: SPIN WAVE THEORY FOR AN HELICAL MAGNET}

For a general Heisenberg model

$$
\mathcal{H}=\sum_{i j} J_{i j} \vec{S}_{i} \cdot \vec{S}_{j}
$$

a classical helical configuration $\vec{S}_{i}=S\left(\hat{u} \sin \left(\vec{Q} \cdot \vec{R}_{i}\right)+\right.$ $\left.\hat{v} \cos \left(\vec{Q} \cdot \vec{R}_{i}\right)\right)$, with $\hat{u} \cdot \hat{v}=0$, has an energy $E_{c l}=$ $N S^{2} J(\vec{Q})$. This helical state lies in the plane normal to the "twist" vector $\hat{n}=\hat{u} \times \hat{v}$. The classical ground state is the helix with pitch $\vec{Q}_{0, c l}$ obtained by minimizing $E_{c l}$, i.e.: $\vec{\partial}_{\vec{Q}} J\left(\vec{Q}_{0, c l}\right)=0$. To perform a spin wave expansion about this classical state we rotate the local quantization axis through an angle $\theta_{i}=\vec{Q} \cdot \vec{R}_{i}$ about the twist vector $\hat{n}$, transforming to a twisted reference frame in which the ordered state is ferromagnetically aligned [36, 37]:

$$
\vec{S}_{i} \rightarrow e^{\vec{A}_{i} \times} \vec{S}_{i}=e^{\vec{Q} \cdot \vec{R}_{i} \hat{n} \times} \vec{S}_{i}
$$

with this prescription, we obtain the following Hamiltonian:

$$
\mathcal{H}^{\prime}=\sum_{i j} J_{i j}\left\{\gamma_{i j}^{+} \vec{S}_{i} \cdot \vec{S}_{j}+\gamma_{i j}^{-}\left(2 \vec{S}_{i} \cdot \hat{n} \vec{S}_{j} \cdot \hat{n}-\vec{S}_{i} \cdot \vec{S}_{j}\right)-\sin \left(\vec{Q} \cdot \vec{R}_{i j}\right) \hat{n} \cdot\left(\vec{S}_{i} \times \vec{S}_{j}\right)\right\}
$$

where $\gamma_{i j}=\frac{1}{2}\left(1 \pm \cos \left(\vec{Q} \cdot \vec{R}_{i j}\right)\right)$. We choose $\hat{n}=\hat{z}$ to carry out the calculations of this section.

The usual way to deal with these magnetic models is to decompose the spins into Holstein-Primakoff (HP) bosons [38, 39 and treat the resulting Hamiltonian as a dilute gas of bosons [40]. We use this representation to calculate LSWT properties of the different spin configurations we are dealing with. The ground state energy of the helicoidal $\vec{Q}=(q, q)$ state is

$$
\begin{aligned}
\frac{E_{0}}{N} & =\frac{S(S+1)}{2} J_{Q_{0}}+\frac{S}{2} \sum_{k} E(k) \\
E(k) & =\sqrt{\left(J_{Q_{0}}-J_{k}\right)\left(J_{Q_{0}}-\frac{1}{2}\left(J_{k+Q_{0}}+J_{k-Q_{0}}\right)\right)}=\sqrt{\left(J_{Q_{0}}-J_{k}\right)\left(J_{Q_{0}}-J_{ \pm}\right)}
\end{aligned}
$$

where again $\overrightarrow{Q_{0}}$ is determined by minimizing $E_{0}$. We can expand $\vec{Q}_{0}$ in powers of $1 / \mathrm{S}$ about its classical value $\left(q_{0, c l}=-\frac{1}{2 J_{2}+4 J_{3}}\right.$ for the state $\left.\overrightarrow{Q_{0}}=\left(q_{0}, q_{0}\right)\right)$. The result is

$$
q_{0}^{i}-q_{0, c l}^{i}=\Delta q_{0}^{i}=\frac{1}{2 S \partial_{i i} J_{Q}} \sum_{k} \partial_{i} J_{ \pm}\left(\frac{J_{k}-J_{Q}}{J_{ \pm}-J_{Q}}\right)^{1 / 2}+O\left(\frac{1}{S^{2}}\right)
$$

The spin stiffness is defined as the second derivative of the free energy with respect to a uniform twist to the 
direction of the order parameter 41. This is equivalent to the response function to a gauge field $\vec{A}_{i}=\delta \vec{Q} \cdot \vec{R}_{i} \hat{k}$ applied to the system, where $\hat{k}$ is the axis of the twist and $\delta \vec{Q}$, its wavevector. Then,

$$
\rho_{i j}^{a}=\frac{\partial^{2} F}{\partial A_{i}^{a} \partial A_{j}^{a}}
$$

For an helical magnet, with $\hat{n} \| \hat{z}$, we have $\rho_{i i}^{x}=\rho_{i i}^{y} \neq \rho_{i i}^{z}$ and $\rho_{x x}^{a}=\rho_{y y}^{a}$. For the purpose of this paper, it will be enough to calculate $\rho_{i i}^{z}$. We obtain:

$$
\begin{aligned}
\rho_{i i}^{z}= & \frac{S^{2} \partial_{i i} J_{Q_{0, c l}}}{2} \\
& +\frac{S}{2}\left\{\partial_{i i} J_{q_{0, c l}}-\left(\sin \left(q_{0, c l}\right)+8 J_{3} \sin \left(2 Q_{c l}\right)\right) S \Delta q_{0}^{i}\right. \\
& \left.+\frac{1}{2} \sum_{k} \frac{\left(2 J_{Q_{0, c l}}-J_{k}-J_{ \pm}\right) \partial_{i i} J_{Q_{0, c l}}-\left(J_{Q_{0, c l}}-J_{k}\right) \partial_{i i} J_{ \pm}}{E(k)}\right\}+O(1)
\end{aligned}
$$

On the other hand, for a bipartite lattice, and when the ground state is Neel ordered $(\pi, \pi)$, it proves more advantageous to use a Dyson-Maleev (DM) decomposition of the spins [38], because then, the Hamiltonian doesn't have terms higher than fourth order and because corrections to mean field results are less important than the ones obtained with HP bosons. The results obtained with this method are identical to those of HP at the mean field level as we will show 40 .

We want to calculate the staggered magnetization for the Neel state of the two models to next to LSWT order in $1 / S$ using DM to check the convergence of the series. The Hamiltonian is given by equation A2, with $\vec{Q}=(\pi, \pi)$. We perform a Fourier transform after introducing DM bosons and rewrite it as:

$$
\begin{aligned}
\mathcal{H}= & S z \sum_{k}\left\{A(k)\left(a_{k}^{+} a_{k}+b_{k}^{+} b_{k}\right)+J_{1} \gamma_{k}\left(a_{k} b_{-k}+a_{k}^{+} b_{-k}^{+}\right)\right\} \\
& -\frac{z J_{1}}{N} \sum_{1234} \delta(1+2-3-4)\left\{2 \gamma_{1+2} a_{1}^{+} a_{-2} b_{-3}^{+} b_{4}+\gamma_{1} a_{1}^{+} b_{2}^{+} b_{-3}^{+} b_{4}+\gamma_{4} a_{1}^{+} a_{-2}^{+} a_{3} b_{4}\right\} \\
& +\frac{z J_{\delta}}{N} \sum_{1234} \delta(1+2-3-4)\left\{\left(\Gamma_{2}^{\delta}-\Gamma_{2-4}^{\delta}\right) a_{1}^{+} a_{2}^{+} a_{3}^{+} a_{4}+\left(\Gamma_{4}^{\delta}-\Gamma_{2-4}^{\delta}\right) b_{1}^{+} b_{2}^{+} b_{3} b_{4}\right\}
\end{aligned}
$$

where

$$
\begin{aligned}
A(k) & =J_{1}-J_{\delta}\left(1-\Gamma_{k}^{\delta}\right) \\
\gamma_{k} & =\frac{1}{2}\left(\cos \left(k_{x} a\right)+\cos \left(k_{y} a\right)\right) \\
\Gamma_{k}^{2} & =\cos \left(k_{x} a\right) \cos \left(k_{y} a\right) \\
\Gamma_{k}^{3} & =\gamma_{2 k}
\end{aligned}
$$

and $\mathrm{z}$ is the coordination number.

We introduce a Bogoliubov transformation, $a_{k}^{+}=$ $u \alpha_{k}^{+}+v \beta_{-k}$ and $b_{-k}=v \alpha_{k}^{+}+u \beta_{-k}$, and normal-order all terms of the Hamiltonian with respect to the vacuum of $\alpha$ and $\beta$, neglect the contribution coming from 
normal-ordered terms involving four Bogoliubov bosons and end up with a quadratic diagonal Hamiltonian. The procedure is standard [42]. We obtain a self-consistent equation for the Bogoliubov amplitude $\mathcal{V}_{k}$, by requiring that the terms proportional to $\alpha^{+} \beta^{+}$and $\alpha \beta$ are zero:

$$
\begin{aligned}
\mathcal{V}(k) & =-\frac{\operatorname{sgn}\left(F_{2}(k)\right)}{\sqrt{2}}\left\{\frac{F_{1}(k)}{\mathcal{E}(k)}-1\right\}^{1 / 2} \\
F_{1}(k) & =A(k) S-\frac{2}{N} \sum_{k^{\prime}}\left\{\mathcal{V}^{2}\left(k^{\prime}\right)\left(1-J_{2}\left(1-\Gamma_{k}^{\delta}-\Gamma_{k^{\prime}}^{\delta}+\Gamma_{k-k^{\prime}}^{\delta}\right)\right)+\gamma_{k^{\prime}} \mathcal{U}\left(k^{\prime}\right) \mathcal{V}\left(k^{\prime}\right)\right\} \\
F_{2}(k) & =\gamma_{k} S+\frac{2}{N} \sum_{k^{\prime}}\left\{\gamma_{k} \mathcal{V}\left(k^{\prime}\right)^{2}+\gamma_{k+k^{\prime}} \mathcal{U}\left(k^{\prime}\right) \mathcal{V}\left(k^{\prime}\right)\right\} \\
\mathcal{E}(k) & =\sqrt{F_{1}^{2}(k)-F_{2}^{2}(k)}
\end{aligned}
$$

here $\mathcal{E}(k)$ is the energy of the spin waves and we have used units where $J_{1}=1$. This expressions can be expanded in powers of $1 / S$. Doing so we obtain the following relation for the staggered magnetization in terms of LSWT functions:

$$
<S^{z}>\simeq S+\frac{1}{2}-\frac{1}{N} \sum_{k} \frac{A(k)}{E(k)}-\frac{J_{\delta}}{N^{2} S} \sum_{k} \frac{\gamma_{k}^{2}\left(1-\Gamma_{k}^{\delta}\right)}{E^{3}(k)} \sum_{k^{\prime}} \frac{A\left(k^{\prime}\right) \Gamma_{k^{\prime}}^{\delta}-\gamma_{k^{\prime}}^{2}}{E\left(k^{\prime}\right)}
$$

where

$$
v(k)^{2}=\frac{1}{2}\left\{\frac{A(k)}{E(k)}-1\right\} \quad E(k)=\sqrt{A^{2}(k)-\gamma_{k}^{2}}
$$

$E(k)$ is the energy of the spin waves in LSWT. We note here that equation A9 agrees with the expression for the staggered magnetization obtained in reference 2 .

We can also compute $\mathcal{E}(k)$ to next to LSWT order in $1 / S$ in the long wavelength limit in terms of the spin wave velocity

$$
\begin{array}{rlrl}
c^{2}(S) & \simeq 8 J_{1} S a\left\{\rho_{0}\left(1+\frac{2}{S}\left(1+\alpha_{1}-\alpha_{4}\right)\right)-\frac{2 J_{2}}{S}\left(\alpha_{2}-\alpha_{1}\right)\right\} & \left(J_{3}=0\right) \\
& \simeq 8 J_{1} S a\left\{\rho_{0}\left(1+\frac{2}{S}\left(1+\alpha_{1}-\alpha_{4}\right)\right)-\frac{4 J_{3}}{S}\left(\alpha_{3}-\alpha_{1}\right)\right\} & \left(J_{2}=0\right) \\
\alpha_{1} & =\sum_{k} \frac{\gamma_{k}^{2}}{E(k)} \quad \alpha_{2}=\sum_{k} \frac{A(k) \Gamma_{k}^{2}}{E(k)} & \\
\alpha_{3} & =\sum_{k} \frac{A(k) \Gamma_{k}^{3}}{E(k)} \quad \alpha_{4}=\sum_{k} \frac{A(k)}{E(k)}
\end{array}
$$

\section{APPENDIX B: SELFCONSISTENT EQUATIONS FOR SCHWINGER BOSONS MEAN FIELD THEORY}

Some years ago, Arovas and Auerbach 43 introduced a $S U(N)$ functional integral expansion as a useful tool to deal with unfrustrated magnets which are disordered due to quantum or thermal fluctuations. It was based on a $S U(N)$ generalization of the spin decomposition 


$$
\vec{S}_{i}=\frac{1}{2} \mathbf{b}_{i}^{+} \vec{\sigma} \mathbf{b}_{i} \quad \mathbf{b}_{i}^{+} \mathbf{b}_{i}=2 S \quad \mathbf{b}_{i}^{+}=\left(b_{i \uparrow}^{+}, b_{i \downarrow}^{+}\right)
$$

originally due to Schwinger [38]. We will use here the generalization to frustrated magnets due to Coleman and coworkers 24, 18] for $S U(2)$.

We begin by rotating the local quantization axis an angle $\theta_{i}=\vec{Q} \cdot \vec{R}_{i}$ so that in the twisted reference frame the spins are ferromagnetically aligned, and introduce the triplet Cooper and singlet particle-hole even-parity fields

$$
\vec{B}_{i j}^{+}=i \mathbf{b}_{i}^{+} \vec{\sigma} \mathbf{b}_{j}^{+} \quad D_{i j}^{+}=\mathbf{b}_{i}^{+} \mathbf{b}_{j}
$$

with which, the action reads (choosing $\hat{n}=\hat{z}$ ):

$$
\begin{aligned}
\mathcal{S} & =\int_{0}^{\beta} d \tau\left\{\mathbf{b}^{+} \partial_{\tau} \mathbf{b}-2 S N \lambda-\frac{N S^{2} J_{Q}}{2}+\lambda D+\frac{1}{4} D J^{+} D-\frac{1}{4} B^{z,+} J^{-} B^{z}\right\} \\
J_{k k^{\prime}}^{ \pm} & =\frac{1}{2}\left\{J_{k-k^{\prime}} \pm \frac{1}{2}\left(J_{k-k^{\prime}+Q}+J_{k-k^{\prime}-Q}\right)\right\}
\end{aligned}
$$

where summation over $\mathrm{k}, \mathrm{k}^{\prime}$ is understood, and $\lambda_{i}(\tau)$ is a Lagrange multiplier which enforces the constraint at all sites and times, and which can be regarded as another field.

We perform a Hubbard-Stratonovich transformation from $D_{k}, B_{k}$ to the bond fields $h_{k}, \Delta_{k}$ and make a saddlepoint approximation of the resulting action in those bond fields. At the saddle-point, they are time independent, spatially uniform and real (and so is the field $\lambda$ ). After diagonalizing the resulting mean field Hamiltonian with a Bogoliubov transformation $b_{k \sigma}=u \gamma_{k \sigma}+v \gamma_{-k-\sigma}^{+}$we find the following Free Energy

$$
\begin{aligned}
\frac{F}{N} & =-(2 S+1) \lambda-\frac{S^{2} J_{Q}}{2}-h_{k} J_{k k^{\prime}}^{+,-1} h_{k^{\prime}}+\Delta_{k} J_{k k^{\prime}}^{-,-1} \Delta_{k^{\prime}}+\frac{2}{\beta} \ln \left(2 \sinh \frac{\beta \epsilon(k)}{2}\right) \\
\epsilon(k) & =\sqrt{(h(k)+\lambda)^{2}-\Delta^{2}(k)}
\end{aligned}
$$

When the ground state is ordered, we have to allow for a one particle condensate contribution to the mean field equations [44, 45, 18]. Besides, the gap in the spin wave spectrum closes -we have a Goldstone mode-, so that we have to adjust $\lambda$ to fulfill this requirement. The saddle point of $\mathrm{F}$ at $T=0$ is given by the following equations:

$$
\begin{aligned}
\frac{F}{N} & =-(2 S+1) \lambda-\frac{S^{2} J_{Q}}{2}-\sum_{k k^{\prime}} \alpha_{k} J_{k k^{\prime}}^{+} \alpha_{k^{\prime}}+\sum_{k k^{\prime}} \beta_{k} J_{k k^{\prime}}^{-} \beta_{k^{\prime}} \\
S+\frac{1}{2} & =S^{*} \delta_{k 0}+\sum_{k} \frac{h_{k}+\lambda}{2 \epsilon(k)} \\
\alpha_{k} & =S^{*} \delta_{k 0}+\frac{h_{k}+\lambda}{2 \epsilon(k)} \\
\beta_{k} & =S^{*} \delta_{k 0}+\frac{\Delta_{k}}{2 \epsilon(k)} \\
\epsilon(k) & =\sqrt{\left(h_{k}-\lambda\right)^{2}-\Delta^{2}(k)}
\end{aligned}
$$

In general, the set of values of the pitch $\vec{Q}$ for the ground state are obtained by minimizing $\mathrm{F}$ with respect 
to $\vec{Q}$. We can obtain $\mathrm{F}$ as a series in $1 / S$ by iterating the mean field equations. We find

$$
\begin{aligned}
\epsilon(k) & =S \sqrt{\left(J_{Q}-J_{k}\right)\left(J_{Q}-\frac{1}{2}\left(J_{k+Q}+J_{k-Q}\right)\right)} \\
\frac{F}{N} & =\frac{S^{2} J_{Q}}{2}+S J_{Q}+\sum_{k} \epsilon(k)=F_{0} S^{2}+F_{1} S
\end{aligned}
$$

The expression for the spin wave energy coincides with that given by LSWT while $F_{1}$ is twice the value given by LSWT (as already stated by Arovas and Auerbach [43] for the unfrustrated case, SBMFT overcounts the number of degrees of freedom).

SBMFT has two major problems in dealing with helicoidal states: (1) Symmetry considerations require the existence of Goldstone bosons at $(0,0)$ and $(q, q)$. We can adjust $\lambda$ so that we have one of them, say $(0,0)$. But then, we don't have the other one: $\left|h_{Q}+\Delta_{0}-h_{0}\right| \neq\left|\Delta_{Q}\right|$ (for Neel and collinear states we do get both zero modes). This leads to unphysical results for the stability diagram of the helicoidal phase, and in particular, to a first order phase transition. (2) We have already shown that $F_{1}$ the contribution of order $\mathrm{S}$ to the free energy is twice the correct value. This means that the pitch $\vec{Q}$ obtained in the minimization is not the correct one.

\section{APPENDIX C: DERIVATION OF THE SCALING EQUATIONS FOR THE LONG WAVELENGTH ACTION}

In this appendix we will use the momentum-shell method to derive recursion relations for the S-matrix of the hydrodynamic action of the $J_{1}-J_{2}-J_{3}$ model, equation 15. This is a generalized $O(3) / U(1)=S^{2}$ model. The unit vector $\hat{n}=(\hat{\pi}, \sigma) ; \hat{\pi}$ is a $n-1=2$-component vector, and $\sigma$ is the direction of quantization. The Smatrix is given by

$$
\mathbf{S}(-\infty, \infty)=\int \mathcal{D} \hat{n} \prod_{x, t} \delta\left(\sigma^{2}(\vec{x}, t)+\hat{\pi}^{2}(\vec{x}, t)-1\right) e^{i \mathcal{S}}
$$

It is convenient to define the dimensionless variables $\tilde{r}=$ $r / a$ and $\tilde{t}=c t / a$ so that the action is

$$
\begin{aligned}
\mathcal{S}= & \left.\frac{1}{2 g_{0}} \int_{-\infty}^{\infty} \int_{1}^{L} d \tilde{t} d^{2} \tilde{r}\left\{\left(\partial_{\tilde{t} \hat{n}}\right)^{2}-\left(\left(\partial_{\tilde{x}} \hat{n}\right)^{2}\right)+\left(\partial_{\tilde{y}} \hat{n}\right)^{2}\right)\right) \\
& +\frac{\sigma_{1}^{0}}{12 \rho(S)}\left(\left(\partial_{\tilde{x} \tilde{x} \hat{n}}\right)^{2}+\left(\partial_{\tilde{y} \tilde{y} \hat{n}} \hat{2}\right)-\frac{\sigma_{2}^{0}}{\rho(S)}\left(\partial_{\tilde{x} \tilde{x} \hat{n}}\right)\left(\partial_{\tilde{y} \tilde{y}} \hat{n}\right)+\frac{2 H S n^{z}}{\rho(S)}\right\}
\end{aligned}
$$

We integrate $\sigma(\vec{x}, t)$ now, and generate an action which is nonlinear in the fields $\hat{\pi}(\vec{x}, t)$. The nonlinear terms constitute the interaction [34]. As we are interested in one loop corrections to the charge g, we retain only the quartic terms. We divide the action now into a free part and the interaction 


$$
\begin{aligned}
\mathcal{S}_{0}= & \frac{1}{2 g_{0}} \int \frac{d w d^{2} k}{(2 \pi)^{2}} \hat{\pi}(k, w) \cdot \hat{\pi}(-k,-w) \\
& \times\left\{w^{2}-k^{2}-g_{0} h+\frac{\sigma_{1}}{12 \rho(S)}\left(k_{x}^{4}+k_{y}^{2}\right)-\frac{\sigma_{2}}{\rho(S)} k_{x}^{2} k_{y}^{2}\right\} \\
\mathcal{S}_{1}= & \left(\prod_{i=1-4} \int \frac{d w_{i} d k_{i}^{2}}{\left(2 \pi^{2}\right)}\right) \hat{\pi}(1) \cdot \hat{\pi}(2) \hat{\pi}(3) \cdot \hat{\pi}(4) \delta^{3}(1+2+3+4) \\
& \times\left\{w_{2} w_{4}-k_{2} \cdot k_{4}-\frac{g_{0} h}{4}+\frac{\sigma_{1}}{12 \rho(S)}\left(k_{x 1} k_{x 2} k_{x 3} k_{x 4}+k_{x 2}^{2} k_{x 4}^{2}+2 k_{x 1} k_{x 2} k_{x 4}^{2}+(x \leftrightarrow y)\right)\right. \\
& \left.-\frac{\sigma_{2}}{\rho(S)}\left(k_{x 1} k_{x 2} k_{y 3} k_{y 4}+k_{x 2}^{2} k_{y 4}^{2}+2 k_{x 1} k_{x 2} k_{y 4}\right)\right\}+\frac{i}{2 \Lambda_{t}} \int \frac{d w d^{2} k}{(2 \pi)^{2}} \hat{\pi}(k, w) \cdot \hat{\pi}(-k,-w)
\end{aligned}
$$

where $h=\frac{a^{3} S H}{c}$ and $\Lambda_{t}$ is an ultraviolet cutoff for the time direction. The free action defines an unperturbed propagator $\mathcal{G}_{0}$, which we use to compute the different terms which arise in the loop expansion. We compute the renormalization of the free term to one loop order, by expanding $e^{S_{1}}$, contracting fields and exponentianting again. The measure cancels the ultraviolet divergent terms arising in the one loop expansion as it does for the classical case. In particular, the regulator $\Lambda_{t}$ doesn't appear in the renormalized theory. The procedure is very well explained in reference 30 , so we refer the reader to it for more details. We obtain

$$
\begin{aligned}
\mathcal{S}_{0}^{\prime}= & \int \frac{d w}{2 \pi} \int_{0}^{e^{-l}} \frac{d^{2} k}{(2 \pi)^{2}} \hat{\pi}(k, w) \cdot \hat{\pi}(-k,-w) \\
& \times\left\{w^{2}\left(1+g_{0} I\right)-k^{2}\left(1+g_{0} I\left(1-\frac{\sigma_{1}-2 \sigma_{2}}{4 \rho(S)}\right)\right)+\frac{\sigma_{1}}{12 \rho(S)}\left(k_{x}^{4}+k_{y}^{4}\right)\left(1+g_{0} I\right)\right. \\
& \left.-\frac{\sigma_{2}}{\rho(S)} k_{x}^{2} k_{y}^{2}\left(1+g_{0} I\right)-g_{0} h\left(1+g_{0} I\right)\right\}
\end{aligned}
$$

where $\mathrm{I}$ is the following loop integral

$$
\begin{aligned}
I & =\int_{-\infty}^{\infty} \frac{d w}{2 \pi} \int_{e^{-l}}^{1} \frac{d^{2} k}{(2 \pi)^{2}} \frac{i}{w^{2}-k^{2}+\frac{\sigma_{1}}{12 \rho(S)}\left(k_{x}^{2}+k_{y}^{2}\right)-\frac{\sigma_{2}}{\rho(S)} k_{x}^{2} k_{y}^{2}} \\
& =\frac{\sqrt{6 \rho(S)} l}{\pi^{2}} \int_{0}^{\pi / 2} \frac{d \theta}{\sqrt{24 \rho(S)-2 \sigma_{1}+\left(\sigma_{1}+6 \sigma_{2}\right) \sin ^{2} \theta}}
\end{aligned}
$$

It diverges at the tricritical point, showing that the scaling analysis can not be applied to dilucidate what happens in the $J_{1}-J_{2}$ model close to the tricritical point.

After performing the rescaling

$$
\begin{aligned}
& k=e^{-l} k^{\prime} \quad w=\frac{c^{\prime}}{c} e^{-l} w^{\prime} \\
& \hat{\pi}=\zeta \hat{\pi}^{\prime} \quad h^{\prime}=\zeta h
\end{aligned}
$$

we find the parameters of the renormalized action in terms of the old ones:

$$
(g h)^{\prime}=e^{2 l}(g h)\left(1+g I \frac{\sigma_{1}-2 \sigma_{2}}{4 \rho(s)}\right)
$$




$$
\begin{aligned}
\left(\frac{1}{g}\right)^{\prime} & =\frac{1}{g} \zeta^{2} e^{-5 l}\left(1+g I\left(1-\frac{3}{8} \frac{\sigma_{1}-2 \sigma_{2}}{\rho(S)}\right)\right) \\
c^{\prime} & =c\left(1-g I \frac{\sigma_{1}-2 \sigma_{2}}{8 \rho(S)}\right) \\
\sigma_{i} & =\sigma_{i}(1-2 l-g I) \\
\rho^{\prime}(S) & =\rho(S)\left(1-g I\left(1+\frac{\sigma_{1}-2 \sigma_{2}}{4 \rho(S)}\right)\right)
\end{aligned}
$$

from which one obtains easily the scaling equations of the model after iterating.

[1] P. W. Anderson, Science 235, 1196 (1987)

[2] S. Chakravarty, B. I. Halperin and D. R. Nelson, Phys. Rev. B39, 2344 (1989)

[3] R. J. Birgueneau, Am. J. Phys.58, 28 (1990); K. Yamada et al, Phys. Rev. B40, 4557 (1989); G. Aeppli et al., Phys. Rev. Lett. 62, 2052 (1989), S. M. Hayden et al., Phys. Rev. Lett. bf 67, 3622 (1991); Y. Uemura et al., Phys. Rev. Lett. 59, 1045 (1987)

[4] J. D. Reger and A. P. Young, Phys. Rev. B37, 5978 (1988); D. A. Huse and V. Elser, Phys. Rev. Lett. 60, 2531 (1988); S. Liang, B. Douçot and P. W. Anderson, Phys. Rev. Lett. 61, 365 (1988), G. Gómez-Santos, J. D. Joannopoulos and J. W. Negele, Phys. Rev. B 39, 4435 (1989); E. Manousakis, Rev. Mod. Phys. 63, 1 (1991)

[5] H. Bethe, Z. Phys. 71, 225 (1931)

[6] F. D. M. Haldane, Phys. Rev. B25, 4295 (1982), Phys. Rev. Lett. 50, 1153 (1983)

[7] I. Affleck, T. Kennedi, E. H. Lieb and H. Tasaki, Comm. Math. Phys. 115, 477 (1988)

[8] P. Chandra and B. Doucot, Phys. Rev. B38, 9335 (1988)

[9] A. Moreo, E. Dagotto, Th. Jolicoeur and J. Riera, Phys. Rev. B42, 6283 (1990)

[10] L. B. Ioffe and A. I. Larkin, Int. J. Mod. Phys. B2, 203 (1988)

[11] A. V. Chubukov, Phys. Rev. B44, 392 (1991)

[12] M. P. Gelfand, R. R. P. Singh and D. A. Huse, Phys. Rev. B40, 10801 (1989), M. P. Gelfand, Phys. Rev. B42, 8206 (1990)

[13] E. Dagotto, I. J. Mod. Phys. B5, 907 (1991)

[14] H. J. Schulz and T. A. L. Ziman, Europhys. Lett. 18, 355 (1992)

[15] T. Einarson and H. Johannesson, Phys. Rev. B43 5867 (1991)

[16] A. F. Barabanov and O. A. Starykh, JETP lett. 51, 311 (1990)

[17] J. H. Xu and C. S. Ting, Phys. Rev. B42, 6861 (1990)

[18] F. Mila, D. Poilblanc and C. Bruder, Phys. Rev. B43, 7891 (1991), C. Bruder and F. Mila, unpublished

[19] L. Bergomi and Th. Jolicoeur, unpublished

[20] S. Sachdev and N. Read, Phys. Rev. Lett. 66, 1773 (1991)

[21] J. Villain, J. Phys. (Paris) 38, 26 (1977)

[22] P. Azaria, B. Delamotte and D. Mouhanna, Phys. Rev. Lett. 68, 1762 (1992) 
[23] T. Dombre and N. Read, Phys. Rev. B39, 6797 (1989)

[24] P. Chandra, P. Coleman and A. I. Larkin, J. Phys.: Condens. Matter 2, 7933 (1990)

[25] F. D. M. Haldane, Phys. Rev. Lett. 50, 1153 (1983); I. Affleck, Nucl. Phys. B265, 409 (1986)

[26] F. D. M. Haldane, Phys. Rev. Lett. 61, 1029 (1988)

[27] T. Dombre and N. Read, Phys. Rev. B38, 7181 (1988); E. Fradkin and M. Stone, Phys. Rev. B38, 7215 (1988)

[28] For a very detailed derivation of the QNL $\sigma \mathrm{M}$, see ref. 15

[29] N. Read and S. Sachdev, Phys. Rev. Lett. 62, 1694 (1989)

[30] A. M. Polyakov, Phys. Lett. 59B, 79 (1975); Gauge Fields and Strings (Harwood, New York 1987)

[31] E. Brézin and J. Zinn-Justin, Phys. Rev. B14, 3110 (1976)

[32] D. R. Nelson and R. A. Pelcovits, Phys. Rev. B16, 2191 (1977)

[33] K. G. Wilson and J. Kogut, Phys. Rep. C12, 75 (1974)

[34] D. J. Amit, Field Theory, the Renormalization Group and Critical Phenomena, 2nd. ed. (World Scientific 1984)

[35] J. A. Hertz, Phys. Rev. B14, 1165 (1976)

[36] E. Rastelli, A. Tassi and L. Reatto, Physica 97B, 1 (1979); E. Rastelli, L. Reatto and A. Tassi, J. Phys. C19, 6623 (1986)

[37] I. Ritchie, PhD Thesis, Cambridge (1991)

[38] D. C. Mattis, The Theory of Magnetism I (Springer-Verlag 1981)

[39] F. J. Dyson, Phys. Rev. 102, 1217 (1956), Phys. Rev 102, 1230 (1956)

[40] A. B. Harris, D. Kumar, B. I. Halperin and P. C. Hohemberg, Phys. Rev. B3, 961 (1971)

[41] J. Rudnick and D. Saslow, Phys. Rev. B16, 2032 (1977)

[42] A. L. Fetter and J. D. Walecka, Quantum Theory of ManyParticle Systems (McGraw-Hill 1971)

[43] D. P. Arovas and A. Auerbach, Phys. Rev. B38, 316 (1988); A. Auerbach and D. P. Arovas, Phys. Rev. Lett. 61, 617 (1988)

[44] P. Nozières and D. Saint James, J. Physique 43, 1133 (1982)

[45] S. Sarker, C. Jayaprakash, H. R. Krishnamurthy and M. Ma, Phys. Rev. B40, 5028 (1989)

FIG. 1. Classical Phase diagram for the $J_{1}-J_{2}-J_{3}$ model. Thick solid lines indicate a discontinuous transition; dashed lines indicate a continuous one. The thin lines are the results of LSWT for the boundaries of the different ordered states for spin $1 / 2$.

FIG. 2. Phase diagram for the $J_{1}-J_{2}-J_{3}$ model. Dashed lines are LSWT; solid, SBMFT. The SWT $\rho_{\text {Neel }}$ vanishes along the dotted line.

FIG. 3. (a) LSWT correction to $q_{0, c l}$ as defined in equation 8. (b) Classical spin stiffness (solid lines) and LSWT corrections to it (dashed lines). 
FIG. 4. (a) Flow diagram for the QNL $\sigma \mathrm{M}$ in $2+1$ dimensions. The dashed line separates the Renormalized Classical region from the Quantum Disordered one. (b) Flow diagram for the $J_{1}-J_{2}-J_{3}$ model, linking CHN and Ioffe and Larkin analysis. We plot in the y-axis $\rho(S)$, the spin stiffness renormalized by short-wavelength quantum fluctuations. Rotating the figure by $90^{\circ}$ we obtain the phase diagram of SWT.

FIG. 5. (a) Phase diagram for the $J_{1}-J_{2}$ model. Dashed lines are LSWT results, while solid lines are those given by SBMFT. (b) same as before in a logarithmic scale. The cross is the result from numerical diagonalization of small clusters [9] 